Cite this: RSC Advances, 2013, 3, 15254

Received 17th April 2013,

Accepted 18th June 2013

DOI: $10.1039 / c 3 r a 41847 b$

www.rsc.org/advances

\title{
Relation between synthesis conditions, dopant position and charge carriers in aluminium-doped $\mathrm{ZnO}$ nanoparticles $\dagger$
}

\author{
Anke Kelchtermans, ${ }^{a}$ Ken Elen, $\uparrow^{\mathrm{ab}}$ Kevin Schellens, ${ }^{a}$ Bert Conings, ${ }^{c}$ Hanne Damm, $\mathfrak{t}^{\mathrm{a}}$ \\ Hans-Gerhard Boyen, ${ }^{c}$ Jan D'Haen, ${ }^{,}$Peter Adriaensens, ${ }^{d}$ An Hardy: ${ }^{\mathrm{ab}}$ and Marlies \\ K. Van Baelł
}

\begin{abstract}
The ability to achieve an understanding of the correlations between chemical synthesis, doping mechanism and properties of aluminium-doped zinc oxide ( $\mathrm{ZnO}: \mathrm{Al}$ ) nanocrystals is of great importance to evaluate the potential of ZnO:Al nanocrystals as optimal building blocks for solution deposited transparent conductive oxide layers. Two series of Al-doped ZnO nanoparticles were synthesized by solution-based methods under different conditions yielding phase-pure wurtzite ZnO:Al nanocrystals with different morphologies (quasispheres and rods). In both series $80 \%$ of the input $\mathrm{Al}$ is incorporated in the ZnO:Al crystals. Furthermore, ${ }^{27} \mathrm{Al}$ nuclear magnetic resonance demonstrated a 5 times higher tetrahedral Al content for the quasispheres, which can be directly linked to the presence of free charge carriers, as showed with Fourier transform infrared spectroscopy. XPS data show the $\mathrm{Zn}^{2+}$ and $\mathrm{O}^{2-}$ chemical state of the constituting ions. It is demonstrated that an efficient $\mathrm{Al}$ doping, creating charge carriers in the $\mathrm{ZnO}$, can be achieved by controlling the chemical synthesis parameters of the nanoparticles.
\end{abstract}

\section{Introduction}

$\mathrm{ZnO}$ is one of the most important wide-band-gap materials (3.3 $\mathrm{eV}$, at $300 \mathrm{~K}$ ) that has superior electronic and optical properties. ${ }^{1-4}$ As a low cost and non-toxic II-VI semiconductor, its promising applications in thin-film transistors, ${ }^{5-7}$ dyesensitized solar cells, ${ }^{8,9}$ gas sensors, ${ }^{10,11}$ varistors $^{12,13}$ and transparent conductive oxides or $\operatorname{TCOs}^{14,15}$ have initiated intensive research. However, doping may be necessary to enhance its performance in view of electronic and optoelectronic devices. ${ }^{16,17} n$-type doping of $\mathrm{ZnO}$ can be achieved by replacing $\mathrm{Zn}^{2+}$ ions with group III metals such as $\mathrm{Ga}^{3+}, \mathrm{Al}^{3+}$ or $\mathrm{In}^{3+}$. By introducing these higher-valence elements into the $\mathrm{ZnO}$ crystals, donor levels allow promotion of free carriers into the conduction band. In this way, it is possible to obtain visible transparency $(>85 \%)$ and high conductivity (103 S $\mathrm{cm}^{-1}$ ). Both are required before $\mathrm{ZnO}$ :Al can be used as a lower

${ }^{a}$ Hasselt University, Institute for Materials Research, Inorganic and Physical Chemistry, Agoralaan Building D, B-3590 Diepenbeek, Belgium.

E-mail: marlies.vanbael@uhasselt.be; Tel: +32011268307

${ }^{b} I M E C$ vzw, division IMOMEC, Agoralaan Building D, B-3590 Diepenbeek, Belgium ${ }^{c}$ Hasselt University, Institute for Materials Research, Materials Physics, Agoralaan Building D, B-3590 Diepenbeek, Belgium

${ }^{d}$ Hasselt University, Institute for Materials Research, Applied and Analytical Chemistry, Agoralaan Building D, B-3590 Diepenbeek, Belgium

$\dagger$ Electronic supplementary information (ESI) available: The ${ }^{27} \mathrm{Al}-\mathrm{NMR}$ figures for all the samples. See DOI: 10.1039/c3ra41847b

† SIM Flanders, SOPPOM program cost alternative to the traditional TCO, indium tin oxide, through the wider accessibility of its composing elements. ${ }^{18}$ The efficiency of the dopant element depends on its electronegativity and ionic radius but it is also strongly dependent of its crystallographic position in the ZnO lattice. This can be actively steered by the synthesis method. ${ }^{16,19-22} \mathrm{ZnO}$ commonly crystallizes in the hexagonal wurtzite structure. In a hexagonally closed-packed lattice of $\mathrm{O}^{2-}$ ions, half of the tetrahedral holes are occupied by the $\mathrm{Zn}^{2+}$ ions, which have indeed a preference for the tetrahedral geometry. ${ }^{23}$ The other half of the tetrahedral sites and all the octahedral sites are empty, providing further possible dopant sites.

Fig. 1 shows a representation of three possible dopant locations for $\mathrm{Al}$ in $\mathrm{ZnO}$. According to theoretical studies, the tetrahedral geometry is the energetically most favorable

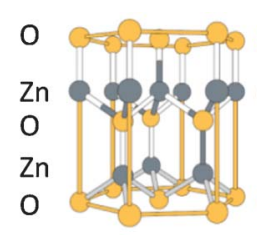

a

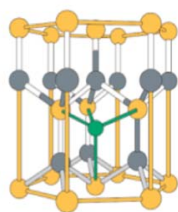

b

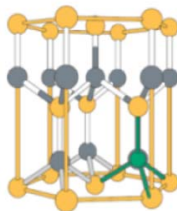

C

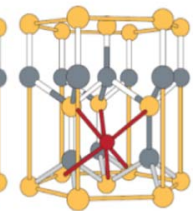

d
Fig. 1 Representation of $\mathrm{ZnO}$ wurtzite crystal structure (a) with $\mathrm{Al}^{3+}$ situated in an empty tetrahedral site (b), substitutional doping (c) and interstitial doping of $\mathrm{Al}^{3+}(\mathrm{d})$. 
situation. ${ }^{24}$ The $\mathrm{Al}^{3+}$ can occupy the empty tetrahedral sites (Fig. 1b) but ideally, the $\mathrm{Al}^{3+}$ ion should substitute a $\mathrm{Zn}^{2+}$ ion in the tetrahedral geometry in order to provide a free electron (charge carrier) which enhances the conductivity (Fig. 1c). ${ }^{25,26}$ Shown in Fig. 1d is the doping mechanism where the $\mathrm{Al}^{3+}$ ion occupies an interstitial octahedrally coordinated site. Despite of the higher formation energy ${ }^{24}$ it is expected that interstitial Al defects may appear when the solubility limit is overcome. ${ }^{27}$ The octahedral coordination, where the $\mathrm{Al}^{3+}$ ion behaves as an acceptor, would decrease conductivity. ${ }^{23,25,28}$

Thus, the $\mathrm{Al}^{3+}$ dopant should be placed in a substitutional, tetrahedral position to effectively modify the electronic properties of the $\mathrm{ZnO}$ host material. Even though the $\mathrm{Al}^{3+}$ ion is smaller than the $\mathrm{Zn}^{2+}$ ion, it has a preference for the octahedral site in most Al-containing oxides. ${ }^{29}$ This different coordination preference of $\mathrm{Al}^{3+}$ and $\mathrm{Zn}^{2+}$ is an important factor that makes substitution still questionable, insisting on further research.

In view of applying $\mathrm{ZnO}: \mathrm{Al}$ as a thin film TCO, alternative, more efficient production technologies are gaining increasing attention compared to current vacuum based techniques. When aiming at a simple and low-cost process with potential for large scale production, wet coating and printing techniques, can offer significant advantages. Two types of chemical solution deposition methods can be distinguished. A first one starts from a molecular precursor, consisting of dissolved precursor molecules or complexes. After deposition, the asdeposited coating is transformed into a dense oxide film upon an appropriate thermal treatment. ${ }^{30}$ In a second method, crystalline oxide nanoparticle building blocks are synthesized first. The resulting particles are then formulated into a suitable ink, followed by their deposition onto the substrate. The fundamental advantage of the latter route is the separation of the heat treatment that is required for the crystallization of the material and the formation of charge carriers, from the process of film formation. A process like this has been successful for coating or printing of ITO, even on flexible substrates (e.g. plastics). ${ }^{31-36}$

A variety of methods such as coprecipitation, ${ }^{37}$ sol-gel, ${ }^{38}$ microwave assisted polyol route, ${ }^{39}$ thermal decomposition synthesis $^{40}$ and hydro/solvothermal synthesis ${ }^{41}$ have been reported for the synthesis of ZnO:Al nanoparticles. For the synthesis of diverse binary metal oxides in general, the benzylalcohol route ${ }^{42}$ is very promising because of its versatile character. To circumvent the drawbacks of commercially unavailable, expensive or undesirable (because of halide impurities) reagents associated with this route, Pinna et al. reported a benzylamine route. ${ }^{43}$ They presented a widely applicable solvothermal route to nanocrystalline iron, indium, gallium and zinc oxide based on the reaction between the corresponding metal acetylacetonate as metal oxide precursor and benzylamine as solvent and reactant. Recently, Baghbanzadeh et $a l .{ }^{44}$ published the synthesis of $\mathrm{ZnO}$ nanoparticles by a combination of the benzylamine route with the benzylalcohol route, wherein the latter combines metal acetylacetonates as metal oxide precursor with benzylalcohol as a solvent and reactant. Because of the versatile character of these routes for $\mathrm{ZnO}$, they were selected as a basis for the current work in order to obtain Al-doped $\mathrm{ZnO}$ nanocrystals.

Though the increased scientific interest in ZnO:Al stimulates its structural and functional exploitation, often less attention is paid to the actual Al-incorporation in these reports. Many reports only mention the nominal Al-percentage, added to the reaction. Nonetheless, it has been shown that there is often an important discrepancy between both values, indicating a non-efficient incorporation in the nanopowder. ${ }^{40}$ Moreover, it can be expected that the performance of ZnO:Al nanocrystals as building blocks for ZnO:Al based TCO layers is sensitive to not only their morphology, size and size distribution but especially to the manner in which the $\mathrm{Al}$ is incorporated.

The aim of the work presented in this paper is to contribute to an understanding of the correlations between chemical synthesis, doping mechanism and properties of Al-doped $\mathrm{ZnO}$ nanocrystals. For this, we focus on solution synthesized Aldoped ZnO nanocrystals, with strongly differing properties, characterized by a range of complementary techniques.

\section{Experimental}

\section{Materials}

Zinc acetylacetonate hydrate $\mathrm{Zn}(\mathrm{acac})_{2} \cdot x \mathrm{H}_{2} \mathrm{O}$, aluminium acetylacetonate $\mathrm{Al}(\mathrm{acac})_{3}(99 \%)$, benzylamine $\mathrm{C}_{6} \mathrm{H}_{5} \mathrm{CH}_{2} \mathrm{NH}_{2}$ and benzylalcohol $\mathrm{C}_{6} \mathrm{H}_{5} \mathrm{CH}_{2} \mathrm{OH}(99 \%)$ are used as received. The amount of hydrated water $(x)$ on zinc acetylacetonate hydrate is verified by high-resolution thermogravimetric analysis (HR-TGA) and inductively coupled plasma atomic emission spectroscopy (ICP-AES) to be 1 per formula unit.

\section{Synthesis nanoparticles - reflux under ambient conditions with benzylamine}

A mixture of $1 \mathrm{~g} \mathrm{Zn}(\mathrm{acac})_{2} \cdot x \mathrm{H}_{2} \mathrm{O}$, appropriate quantities of $\mathrm{Al}(\mathrm{acac})_{3}$ (ranging from 0 at $\%$ to 5 at $\%$, with respect to $\mathrm{Zn}$ ) and $40 \mathrm{ml}$ benzylamine is poured in a three-neck flask and heated to $200{ }^{\circ} \mathrm{C}$. The temperature is kept at $200{ }^{\circ} \mathrm{C}$ for $1 \mathrm{~h}$ while stirring. After cooling down to room temperature, the resulting precipitate is centrifuged and washed thoroughly with water and methanol several times. The precipitate is finally dried in a furnace at $60{ }^{\circ} \mathrm{C}$ in air.

\section{Synthesis nanoparticles - solvothermal synthesis with benzylalcohol}

A mixture of $1 \mathrm{~g} \mathrm{Zn}(\mathrm{acac})_{2} \cdot x \mathrm{H}_{2} \mathrm{O}$, appropriate quantities of $\mathrm{Al}(\mathrm{acac})_{3}$ (ranging from 0 at $\%$ to 5 at $\%$, with respect to $\mathrm{Zn}$ ) and $40 \mathrm{ml}$ benzylalcohol is stirred for $30 \mathrm{~min}$ in a Teflon liner (inner volume of $80 \mathrm{ml}$ ). This Teflon liner is then transferred into a stainless steel vessel which is sealed and placed in a preheated furnace at $200{ }^{\circ} \mathrm{C}$ for $6 \mathrm{~h}$. After cooling down to room temperature, the resulting precipitate is centrifuged and washed thoroughly with water and methanol several times. Finally, the precipitate is dried in a furnace at $60{ }^{\circ} \mathrm{C}$ in air. 


\section{Characterization}

The size and morphology of the $\mathrm{ZnO}(\mathrm{Al})$ nanocrystals were characterized with transmission electron microscope (TEM), carried out on a FEI Tecnai Spirit at an acceleration voltage of $120 \mathrm{kV}$. For this purpose, a small drop of the powdered sample was redispersed in methanol and deposited on a carbon-film coated copper grid and dried under an infrared-lamp. The amount of adsorbed water on zinc acetylacetonate hydrate and the at $\% \mathrm{Al}$ present in the $\mathrm{ZnO}$ powder was determined by inductively coupled plasma atomic emission spectroscopy (ICP-AES) using a PerkinElmer Optima device operating at 3300 V. Sample preparation includes dissolving and subsequent dilution of the nanopowder in $5 \% \mathrm{HNO}_{3}$ (J. T. Baker, 69$70 \%$, Baker Instra-analyzed Reagent) to the extent that the target concentration is situated within the calibration range of 1 to $10 \mathrm{ppm}$. High resolution thermogravimetric analysis (HRTGA) is performed on a 2950 Thermo-gravimetric analyzer of TA Instruments to confirm the amount of adsorbed water on zinc acetylacetonate. The ${ }^{27} \mathrm{Al}$ solid-state magic angle spinning nuclear magnetic resonance (MAS-NMR) measurements were performed at room temperature on an Agilent VNMRS 400 $\mathrm{MHz}$ spectrometer (9.4 $\mathrm{T}$ wide bore magnet) equipped with a Varian-Chemagnetics T3HX $3.2 \mathrm{~mm}$ probe. The resonance frequency of the aluminum nuclei is $104.22 \mathrm{MHz}$. The samples were packed into a $3.2 \mathrm{~mm}$ zirconia rotor and sealed with tight fitting Torlon caps. The spectra were obtained at a rotor spinning rate of $18 \mathrm{kHz}$ by using a single pulse experiment with a $\pi / 2$ pulse of $4.2 \mu \mathrm{s}$, an acquisition time of $10 \mathrm{~ms}$, a preparation delay of $6 \mathrm{~s}$ and a spectral width of $420 \mathrm{kHz}$ (nanospheres) and $100 \mathrm{kHz}$ (nanorods). Depending on the $\mathrm{Al}$ content, the number of accumulations was varied between 10.000-50.000 (spheres) and 4.000-10.000 (rods). The chemical shift scale, in parts per million (ppm), was externally referenced to $\mathrm{AlCl}_{3} \cdot 6 \mathrm{H}_{2} \mathrm{O}$ at $0 \mathrm{ppm}$. The actual tetrahedral and octahedral $\mathrm{Al}$ content in relation to $\mathrm{Zn}$ is calculated by multiplying the actual Al content, determined by ICP-AES, with respectively the tetrahedral and octahedral percentage, obtained by ${ }^{27} \mathrm{Al}-\mathrm{NMR}$ characterization. The crystal phase of the dry nanopowders was determined at room temperature by $\mathrm{X}$-ray diffraction (XRD) in the $2 \theta$ ranges from 30 to $60^{\circ}$, using a Siemens D-5000 diffractometer with $\mathrm{Cu}-\mathrm{K} \alpha 1$ radiation. Before recording the XRD-patterns, $\mathrm{LaB}_{6}$ powder was mixed with the $\mathrm{ZnO}$ :Al powders as an internal standard which permits a correction for systematic errors. The value of the shape factor for spheres in the Debeye-Scherrer formula is 0.90. Fouriertransform infrared (FTIR) spectra were recorded at room temperature using a Vertex 70 FTIR spectrometer from Bruker Optics. The transmittance of KBr-pellets containing $0.5 \mathrm{wt} \%$ $\mathrm{ZnO}$ :(Al) was measured in the wavenumber interval of 4000$400 \mathrm{~cm}^{-1}$. XPS experiments were performed on a Physical Electronics (PHI) 5600LS electron spectrometer equipped with a small-spot X-ray source providing monochromatized Al-K $\alpha$ photons $(1486.6 \mathrm{eV})$. For the high-resolution spectra the overall energy-resolution (photons and photoelectrons) was adjusted to $0.31 \mathrm{eV}$ (FWHM). The binding energy scale was calibrated by means of an independent Au reference sample, setting the $\mathrm{Au}-4 \mathrm{f}_{7 / 2}$ core level position to $84.00 \mathrm{eV}$.
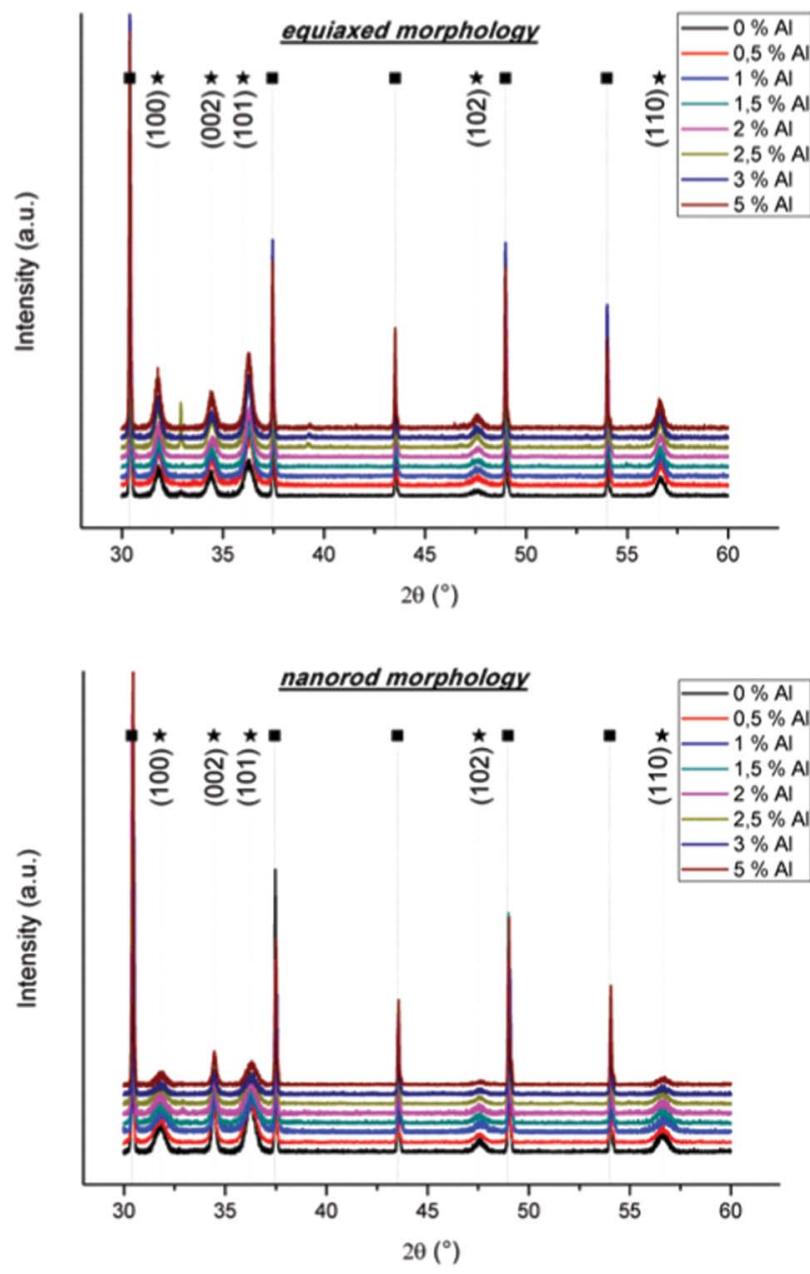

Fig. $2 X R D$ patterns of the equiaxed AZO nanopowders (top) and the AZO nanorod powders (bottom). The $\mathbf{\square}$-symbols correspond with the signals of $\mathrm{LaB}_{6}$, the internal standard and the $\star$-symbols indicate the wurtzite $\mathrm{ZnO}$ structure according to JCPDS card file No 36-1451.

\section{Results and discussion}

Two different synthesis routes towards Al-doped $\mathrm{ZnO}$ crystals were applied. A solvothermal reaction with benzylalcohol as the solvent leads to rod shaped nanoparticles and a reaction under reflux conditions with benzylamine as the solvent yields nano-equiaxed particles. Of both series, ZnO particles with varying $\mathrm{Al}$ content were obtained. All samples of both series of equiaxed particles and nanorod particles exhibit (100), (002), (101), (102) and (110) reflections of the hexagonal wurtzite structure (JCPDS 36-1451) in the XRD patterns (Fig. 2). In addition, no $\mathrm{Al}$ rich secondary phases such as gahnite or alumina are detected within the detection limits of the apparatus (a few percent). The two synthesis routes result in different morphologies as shown in Fig. 3 and 4 . For the $\mathrm{ZnO}$ (Al) powders synthesized under reflux and ambient conditions with benzylamine as the solvent, a uniform equiaxed morphology is revealed (Fig. 3). The quasispherical nanoparticles exhibit diameters ranging between 20 and 30 


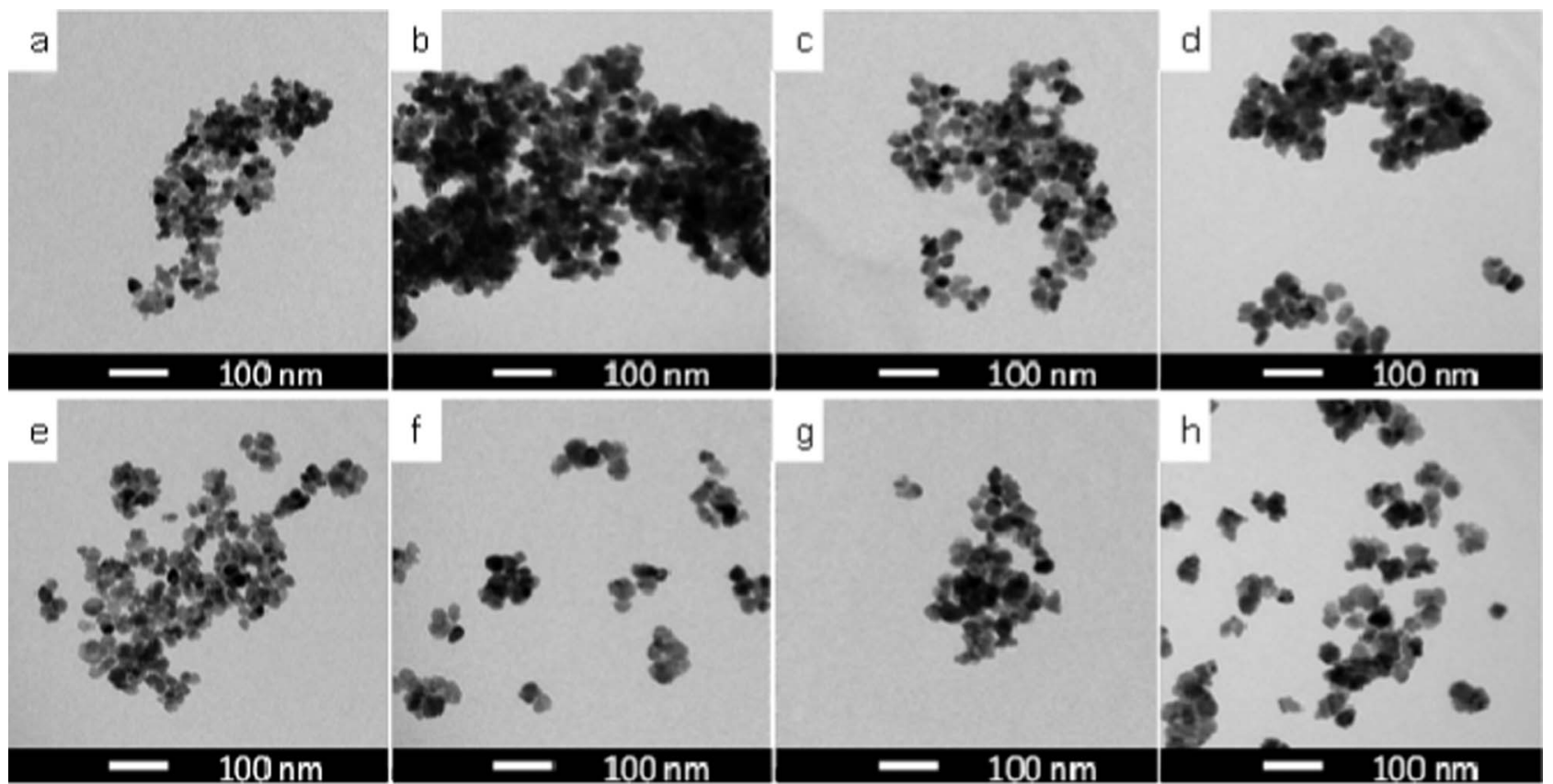

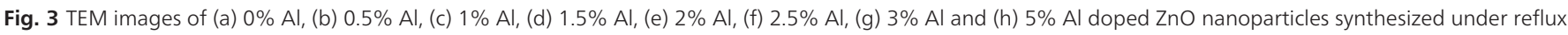
conditions with benzylamine as the solvent.

$\mathrm{nm}$, which are expected to be a beneficial compromise between conductivity and transparency for TCOs. ${ }^{45}$ The nominal Al content in the AZO nanoparticles does not seem to have a significant effect on the average diameter and the size distribution of the nanoparticles. The mean crystallite size of the quasispherical $\mathrm{ZnO}$ (Al) nanoparticles with 0 and 5 at\% $\mathrm{Al}$ was also estimated from the highest intensity peak of the XRD-patterns by the Debye-Scherrer's formula ${ }^{46}$ to be $\sim 19$ and $27 \mathrm{~nm}$, respectively. These crystallite sizes are in the same range as the grain sizes, shown by the TEM-images, implying that the nanoparticles are monocrystalline. The TEM images of the $\mathrm{ZnO}(\mathrm{Al})$ powders obtained from the solvothermal synthesis show a one-dimensional morphology (Fig. 4). This nanorod morphology continues throughout the whole doping series. The average diameter of the rods is $15 \mathrm{~nm}$ for the undoped $\mathrm{ZnO}$ nanocrystals and $10 \mathrm{~nm}$ for the AZO nanocrystals. They all have lengths varying between 90 and $150 \mathrm{~nm}$. By comparison of our experiments with those of e.g. Suwanboon ${ }^{47}$ we confirm that not only the nominal amount of $\mathrm{Al}$ in the $\mathrm{ZnO}$ determines their morphology and other properties but other factors are of importance too. Possibly, the effective Al uptake, which differs from the nominal amount (see further), as well as the mechanism and kinetics of nanoparticle formation, determined by the synthesis conditions, play a key role. To investigate the Al-incorporation in these nanoparticles, first the total amount of $\mathrm{Al}$ incorporated in the $\mathrm{ZnO}$ powder is determined. ICP-AES measurements provide the chemical composition of the thoroughly washed powders which can be compared with the nominal amount of $\mathrm{Al}$ added during the synthesis (Fig. 5).
It was found that for both the nano-equiaxed and the nanorod series, the $\mathrm{Al}^{3+}$ uptake was similar; a linear relation can be observed between the nominal and the actual $\mathrm{Al}$ percentage whereby however the actual $\mathrm{Al}^{3+}$ content is lower than the nominal content. Taking into account an error on the fitting, this linear relation has a proportionality coefficient of approximately 0.8 , which would mean that about $80 \%$ of the $\mathrm{Al}$ added in the synthesis recipe is really taken up during the synthesis of the nanoparticles. Thu et al. ${ }^{40}$ also obtained actual $\mathrm{Al}^{3+}$ contents considerably lower than the nominal ones, although they observed an exponential relation, indicating the influence of the synthesis method. This value however only provides us general information of the aluminium content in the powder. In order to find out how the $80 \%$ actual $\mathrm{Al}$ content is divided over the tetrahedral and octahedral positions, ${ }^{27} \mathrm{Al}-$ NMR can provide information on the local environment of the aluminium ions. All ${ }^{27} \mathrm{Al}-\mathrm{NMR}$ spectra of both nanorods and nanospheres present two signals with a rather narrow linewidth indicating a highly ordered, crystalline environment. This implies that the aluminium is incorporated into the crystalline ZnO lattice during synthesis. ${ }^{48}$ One signal has an isotropic chemical shift in the region of 0-20 ppm, corresponding to the range of $\mathrm{Al}$ coordinated to 6 oxygen atoms (octahedral). The other signal at $75 \pm 15$ ppm is characteristic for aluminium in a 4 -fold coordination with oxygen (tetrahedral). ${ }^{49}$ Taking into account geometry preference rules, one would expect a preference of $\mathrm{Al}$ for the octahedral site ${ }^{29}$ as present in $\alpha-\mathrm{Al}_{2} \mathrm{O}_{3}$. The presence of the aluminium in a 4 -fold coordination can therefore be an indication of an efficient doping. For the nano-equiaxed sample containing 0.5 at $\% \mathrm{Al}$, the fraction of tetrahedral $\mathrm{Al}$ even exceeds the octahedral one 

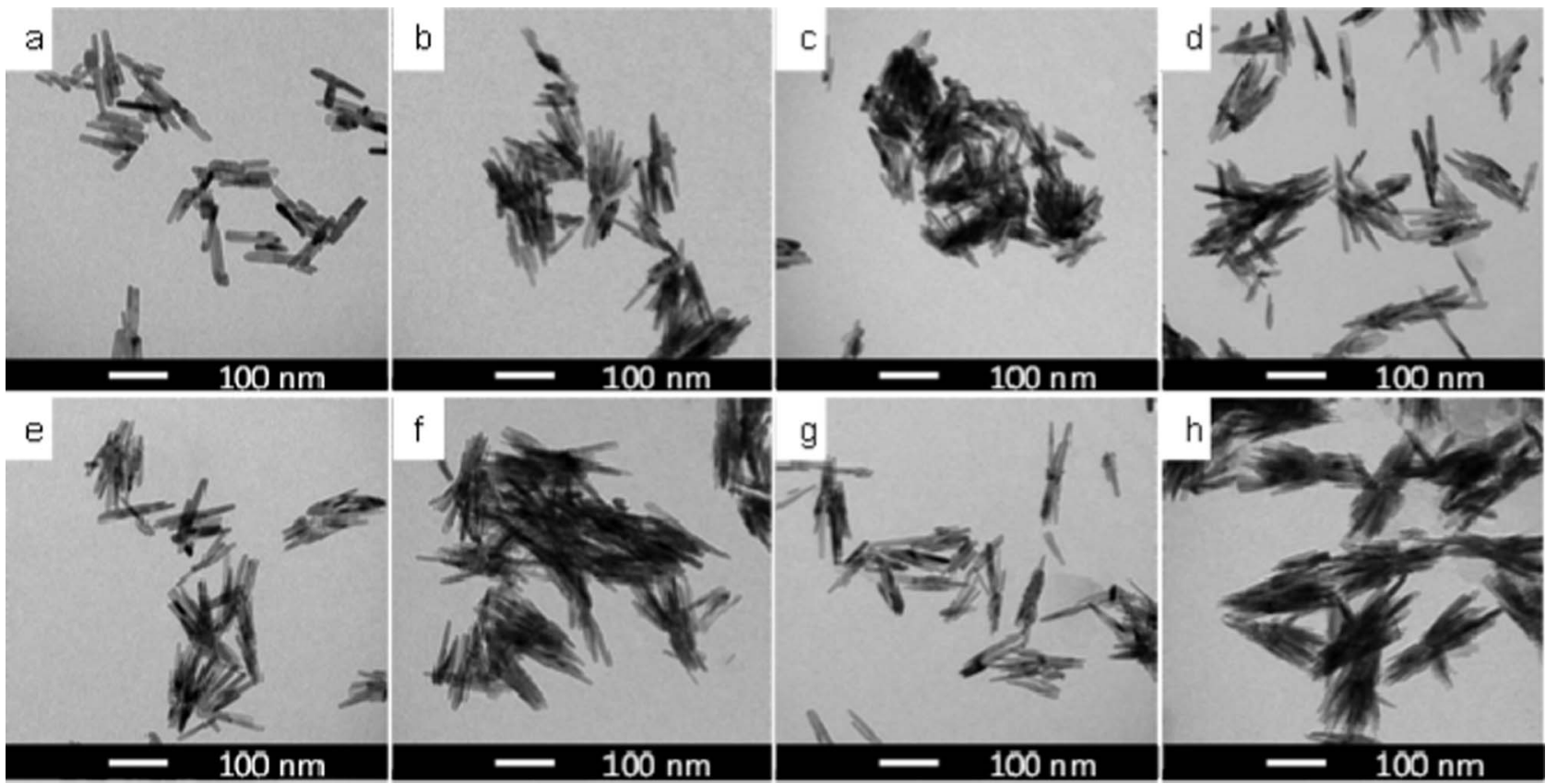

Fig. 4 TEM images of (a) $0 \%$ Al, (b) $0.5 \%$ Al, (c) $1 \%$ Al, (d) $1.5 \%$ Al, (e) $2 \%$ Al, (f) $2.5 \%$ Al, (g) $3 \%$ Al and (h) $5 \%$ Al doped ZnO nanoparticles synthesized under solvothermal conditions with benzylalcohol as the solvent.

(inset Fig. 6a). With higher $\mathrm{Al}$ content, the fraction tetrahedral $\mathrm{Al}$ decreases (ESI $\dagger$ ). Although the ratio of the integrated values of two resonances at their isotropic chemical shifts might not be fully quantitative, the change in relative intensities of these two signals can be used to provide a comparative, semiquantitative estimate of the relative $\mathrm{Al}$ site occupation in the different samples. Combining the ${ }^{27} \mathrm{Al}-\mathrm{NMR}$ data with the ICPAES data gives a realistic idea about the actual tetrahedral and octahedral $\mathrm{Al}$ content in relation to $\mathrm{Zn}$. These recalculated data are displayed in Fig. 6a for the nano-equiaxed series. From a least squares regression fit, it can be concluded that the tetrahedral $\mathrm{Al}$ content is constant, regardless of the total $\mathrm{Al}$

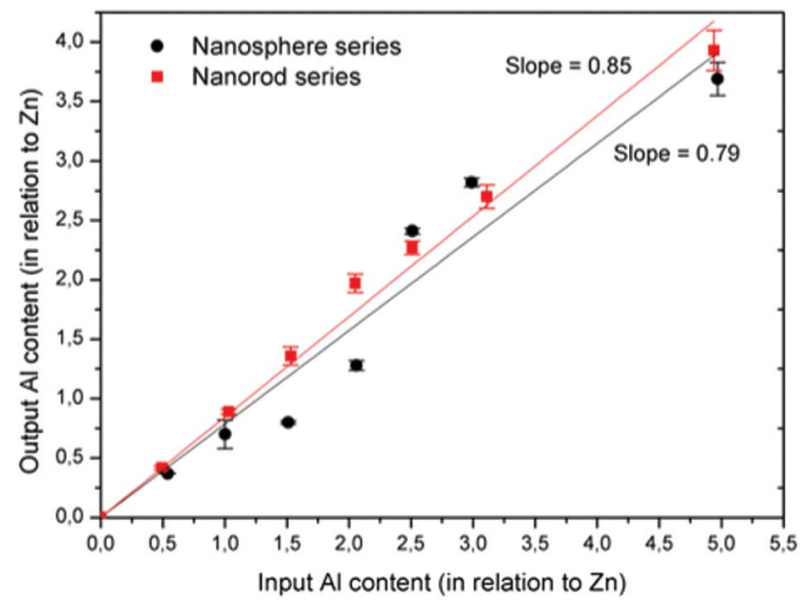

Fig. 5 Linear relationship between Al input content and Al output content for both the nano-equiaxed series and the nanorod series. included in the nanocrystals. The intercept of the fitted trend line reveals that, with respect to the amount of $\mathrm{Zn}, 0.26 \% \mathrm{Al}$ occupies a 4-fold coordination. The remainder of the incorporated $\mathrm{Al}$ is positioned on octahedral sites, as apparent from the linearly increasing amount of octahedral Al. Even though the ${ }^{27} \mathrm{Al}-\mathrm{NMR}$ spectra of the nanorod series show the same Al chemical shifts and also a decreasing trend in the tetrahedral $\mathrm{Al}$ fraction as for the quasispherical series, the intensities corresponding to the aluminium in a 4-fold coordination (75 $\pm 15 \mathrm{ppm})$ are significantly lower (inset Fig. 6b and $\mathrm{ESI} \dagger$ ). The actual tetrahedral $\mathrm{Al}$ content in relation to $\mathrm{Zn}$ shows again a constant value. However, the 5 times lower intercept value (Fig. 6b) indicates that 5 times less $\mathrm{Al}$ can be incorporated in the tetrahedral sites via the solvothermal synthesis route (rod shaped particles) as compared to the synthesis under reflux conditions. The remainder of the $\mathrm{Al}$ taken up by the nanocrystals is located in the octahedral sites. As often reported in literature, the ratio of substitutional and interstitial aluminium can also influence the lattice parameters of the $\mathrm{ZnO}$ wurtzite crystal structure. Because of the difference in size (4-fold coordinated $\mathrm{Zn}^{2+}$ ion $=0.74 \AA$, 4-fold coordinated $\mathrm{Al}^{3+}$ ion $=0.54 \AA$ ), substitutional doping is expected to cause contraction of the lattice parameters. ${ }^{50,51}$

Interstitial doping on the other hand, would be responsible for an expansion of the lattice. ${ }^{38,52,53}$ These effects are reflected by shifts in peak positions in the X-ray diffractograms to higher and lower 2 theta angle respectively. From the XRD patterns (Fig. 2), it appears that no significant shifts can be observed in either of both series. Taking into account the results from the ${ }^{27} \mathrm{Al}-\mathrm{NMR}$ data, revealing the presence of both tetrahedral and octahedral aluminium, they are in accordance 

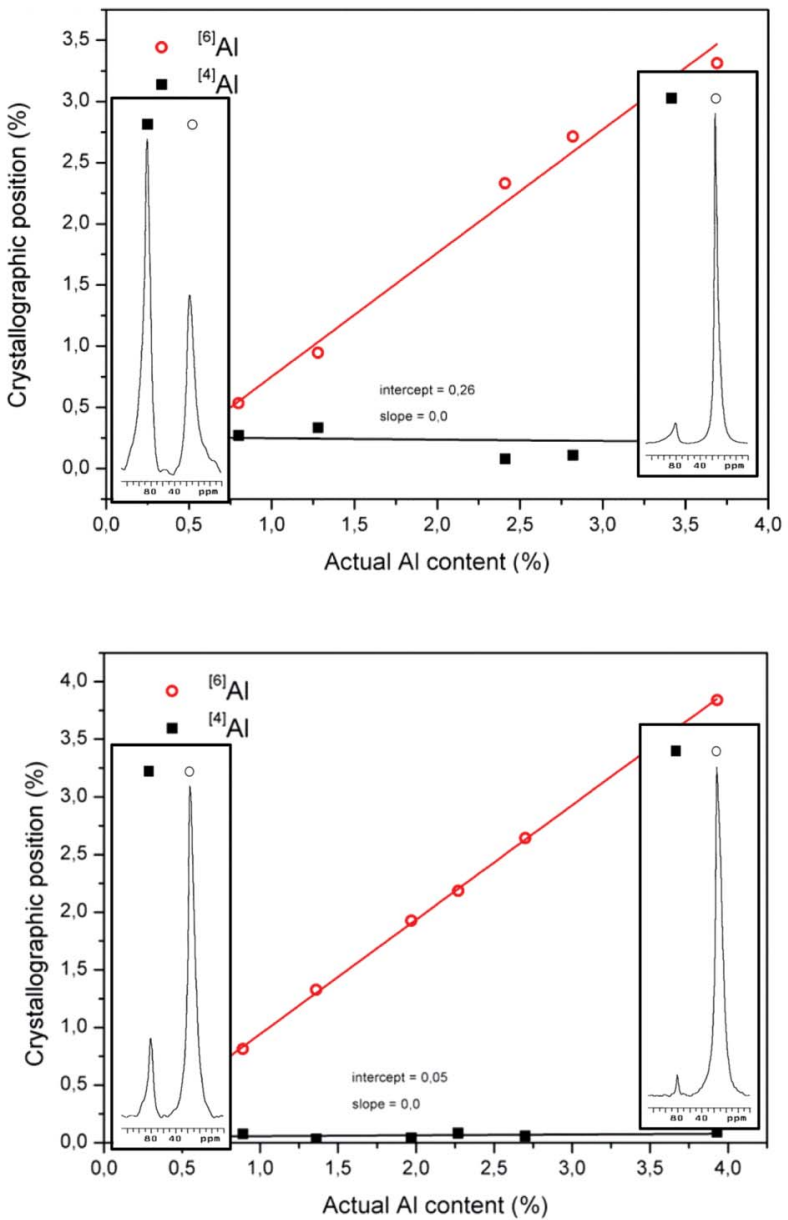

Fig. 6 Percentage ${ }^{[4]} \mathrm{Al}$ and ${ }^{[6]} \mathrm{Al}$ in the nano-equiaxed series (a) and the nanorod series (b). ${ }^{27} \mathrm{Al}-\mathrm{NMR}$ spectra of the 0.5 and $5 \%$ Al-doped $\mathrm{ZnO}$ powders are shown in the insets. -symbols corresspond with tetrahedral Al, $\mathrm{O}$-symbols correspond with octahedral Al.

with the XRD observations if we assume that the tetrahedral Al has taken substitutional positions and the octahedral aluminum has taken interstitial positions. Indeed, as both types of substitutions would cause a diffraction peak shift in opposite direction a complementary compensation is in accordance with expectations.

Even though the ${ }^{27} \mathrm{Al}$-NMR spectra have clearly demonstrated that different amounts of $\mathrm{Al}$ are located at tetrahedral sites in the $\mathrm{ZnO}$ lattice, it is not unambiguously proven that they are substituting $\mathrm{Zn}^{2+}$-ions. It might be possible as well that the tetrahedral $\mathrm{Al}$ is due to $\mathrm{Al}^{3+}$ ions occupying the vacant tetrahedral positions (see Fig. 1b) of the zincite structure instead of substituting a zinc ion (see Fig. 1c). This would be undesirable as it would not contribute to improved conductivity in the material. Therefore, complementary information is obtained from FTIR absorption spectra which can reveal the presence or absence of free charge carriers in the powder. ${ }^{3,39}$ FTIR spectra are recorded of all the powders of the nanoequiaxed as well as the nanorod series to evaluate the presence a)

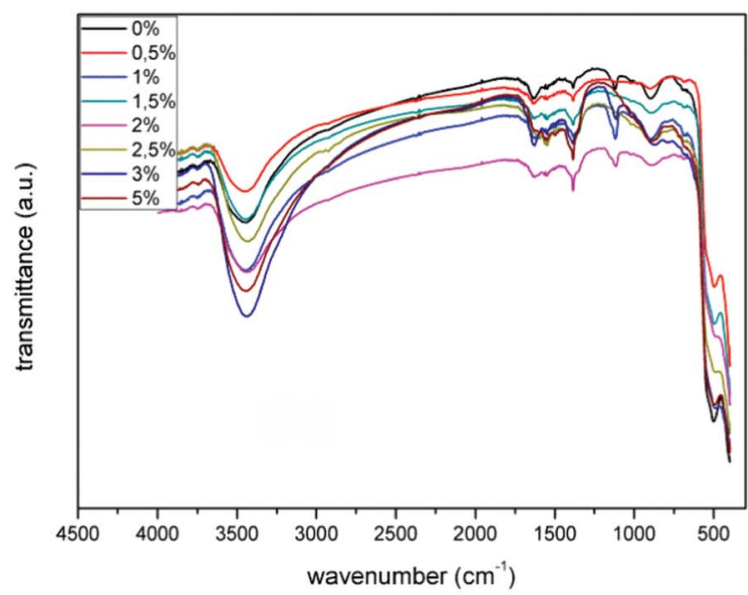

b)

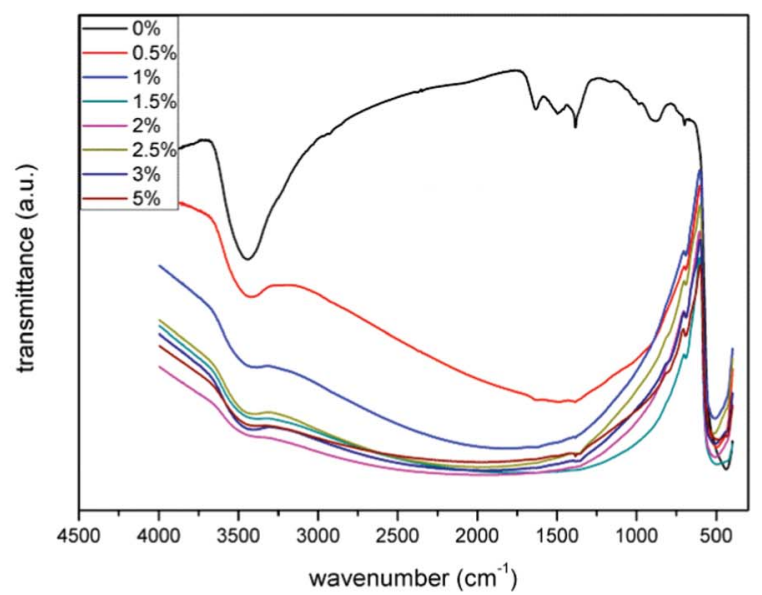

Fig. 7 FTIR spectra of the nanorod series (a) and the nano-equiaxed series (b) as a function of the nominal $\mathrm{Al}$ content.

of free charge carriers indicative for substitutional doping. ${ }^{3,19,39}$

All the spectra of the nanorod series (Al doped as well as pure $\mathrm{ZnO}$ ) exhibit the same features when compared with the FTIR absorption spectrum of undoped ZnO (Fig. 7a). The broad absorption bands at $\sim 3430 \mathrm{~cm}^{-1}$ and $\sim 1645 \mathrm{~cm}^{-1}$ encompass the $\mathrm{O}-\mathrm{H}$ stretching vibrations of adsorbed water on the $\mathrm{ZnO}(: \mathrm{Al})$ surface. The strong absorption band between 500 and $400 \mathrm{~cm}^{-1}$ can be attributed to the stretching modes of $\mathrm{Zn}-\mathrm{O}$ and/or Al-O. The FTIR spectra of the series of nanosphere particles (Fig. 7b) reveal the similar features only for the undoped $\mathrm{ZnO}$ sample. The spectra of the Al-doped $\mathrm{ZnO}$ quasispheres on the other hand exhibit an additional broad absorption band between 4000 and $1500 \mathrm{~cm}^{-1}$, which can be attributed to surface plasmon absorption and related to the presence of free charge carriers. ${ }^{54}$ We therefore infer that the tetrahedrally coordinated $\mathrm{Al}$, incorporated in the spherical particles, has taken substitutional positions and hence has created extra free charge carriers that can increase the conductivity of the material. ${ }^{23}$ Likewise, the absence of the additional broad absorption band in the nanorod shaped particles, with a much lower tetrahedral Al fraction can then 
be explained by the inefficient substitutional doping. In comparison with other findings, ${ }^{23,25}$ these results emphasize the important influence of the synthesis route on both the $\mathrm{Al}$ uptake in the powder and especially the position of the $\mathrm{Al}$ in the $\mathrm{ZnO}$ lattice, which in turn can have a large effect on the further characteristics of the ZnO:Al nanocrystals. Based on these results, the Al-doped $\mathrm{ZnO}$ nanospheres with a theoretical aluminium content of 0.5 at $\%$ will exhibit the best conductive properties. XPS measurements are carried out to determine the stoichiometry and chemical state of the constituent elements. The chemical analysis reveals an $\mathrm{Al}$ content of 0.3 at $\%$ which, however, is at the detection limit of the spectroscopy in this case and, thus, suffers from a large error bar $( \pm 0.2$ at $\%)$. Furthermore, a binding energy of $74.70 \mathrm{eV}$ is obtained for the (unresolvable) Al $2 \mathrm{p}$ core doublet which points towards an oxidation state close to $\mathrm{Al}$ in $\mathrm{Al}_{2} \mathrm{O}_{3}$ ( $\mathrm{Al}$ 2p: $74.60 \mathrm{eV})^{55}$ thereby giving further support for $\mathrm{Al}$ residing at substitutional positions rather than at interstitial sites. The $\mathrm{O}$ $1 \mathrm{~s}$ binding energy region has extensively been studied in the past to identify oxygen deficiencies in Al-doped ZnO. Often, three chemically different components are assumed and attributed to stoichiometric $\mathrm{ZnO}(530.15 \mathrm{eV})$, oxygen-deficient regions $(531.25 \mathrm{eV})$, and chemisorbed oxygen $(532.4 \mathrm{eV}){ }^{56-58}$ Fig. 8 presents the high-resolution $\mathrm{O} 1 \mathrm{~s}$ spectrum acquired on 0.5 at $\%$ ZnO:Al spherical nanocrystals. Clearly, two components can be identified at binding energies of $531.0 \mathrm{eV}$ and $532.8 \mathrm{eV}$ which, according to the studies mentioned before, should be assigned exclusively to oxygen-deficient $\mathrm{ZnO}$ (main peak) and chemisorbed species (shoulder). Such a conclusion, however, is misleading for the following reason: in Fig. 8 spectra have been added taken from a ZnO single crystal after its annealing for $2 \mathrm{~h}$ at $1100{ }^{\circ} \mathrm{C}$ in pure oxygen atmosphere (which certainly removed any oxygen vacancies present before) and its transfer within $15 \mathrm{~s}$ into the electron spectrometer. Clearly, the $\mathrm{O} 1 \mathrm{~s}$ spectrum consists of a main line at a binding energy of $531.0 \mathrm{eV}$ (which perfectly coincides with the strongest line found for the nanocrystals) together with an additional shoulder centered at $532.8 \mathrm{eV}$. This shoulder raises in intensity if the single crystal is exposed to ambient for $5 \mathrm{~min}$ thus indicating an increased adsorption of water from the air and/or the formation of $\mathrm{OH}$ groups at the sample surface. In order to verify the presence of a surface layer, angle-resolved measurements have been performed at $80^{\circ}$ from the surface normal thereby significantly increasing the surface sensitivity of the spectroscopy. Obviously, by changing the photoelectron detection angle from $20^{\circ}$ to $80^{\circ}$ results in a significantly increased spectral intensity at $532.8 \mathrm{eV}$ which identifies this component as purely surface-related. Using the single crystal as a reference, on one hand, a high crystal quality can be concluded for the Al-doped nanocrystals resulting from the coinciding main lines in the $\mathrm{O}$ 1s spectra. On the other hand, the surface of these nanocrystals (which have been exposed to air during/after their synthesis) reveals a similar surface termination as found for the single crystalline sample exposed to ambient. It is worth to mention that, in case of a spherical nanocrystal, a large contribution of photoelectrons emitted
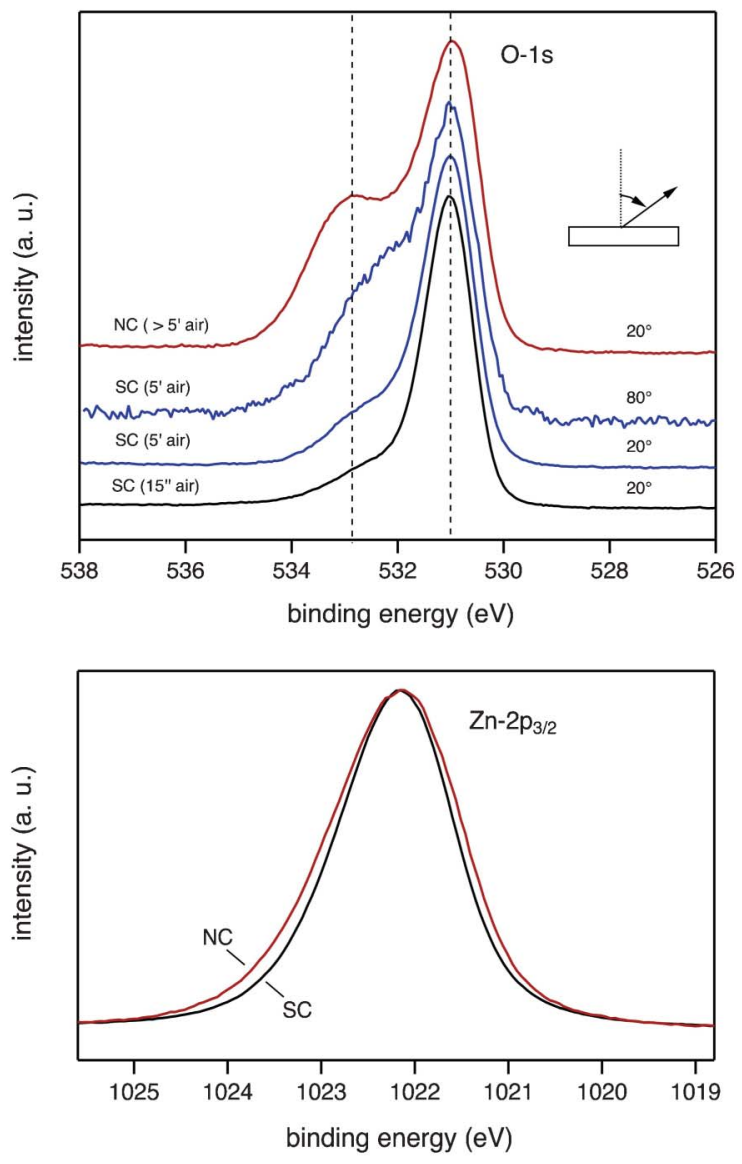

Fig. 8 High-resolution XPS spectra of the O 1s (top) and Zn $2 p_{3 / 2}$ (bottom) core level regions acquired on 0.5 at\% $\mathrm{ZnO}$ :Al spherical nanocrystals (NC) in comparison with results measured on a $\mathrm{ZnO}$ single crystal (SC).

from its surface towards the electron energy analyzer are measured with high surface sensitivity. This is especially true for photoelectrons emitted in the vicinity of the nanocrystal's 'equator'. Hence, a large contribution of surface-related oxygen species to the total $\mathrm{O} 1 \mathrm{~s}$ spectrum can be expected which can indeed be observed in Fig. 8 .

More information about the impact of Al dopants onto the $\mathrm{ZnO}$ matrix can be derived from the high-resolution $\mathrm{Zn} 2 \mathrm{p}_{3 / 2}$ core level spectrum presented in Fig. 8 (bottom panel). Here, the presence of $\mathrm{Al}$ results in a small but significant increase in line width compared to the $\mathrm{ZnO}$ single crystal the latter serving again as a reference system. A line broadening is expected, on one hand, in case of fluctuations in the local coordination with oxygen around the probed $\mathrm{Zn}$ atoms induced by the presence of $\mathrm{Al}$ in the neighbourhood. On the other hand, a line broadening as compared to the single crystal might also reflect a reduced lifetime of the photoionized hole states in the nanocrystals due to an increased density of free electrons (provided by dopants) which finally neutralize the photoionized atoms on the femtosecond scale. 


\section{Conclusions}

It was shown that apparently small changes in synthesis parameters, solvent and pressure, can greatly affect the properties of ZnO:Al nanoparticles. First of all, the morphology may alter from nanospheres to nanorods (TEM), independent of the global $\mathrm{Al}$ content (ICP). The determination of the coordination number of $\mathrm{Al}$, by means of $\mathrm{NMR}$, however elucidates that the fraction of tetrahedral to octahedral coordination, for identical Al dopant concentrations, differs significantly. The tetrahedrally coordinated Al can either be positioned in tetrahedral vacancies of the wurtzite lattice, ineffective for charge carrier generation, or be in substitutional positions, creating charge carriers. Both cases cannot be distinguished by NMR, but FTIR measurements demonstrate the presence of free charge carriers for the $\mathrm{ZnO}: \mathrm{Al}$ nanospheres with high tetrahedral Al content. Hence, at least part of the tetrahedral $\mathrm{Al}$ must be in substitutional positions which is also supported by XPS results. Based on the present study leading to a full understanding of the particle structures, it can now be concluded that in order to obtain efficient doping in ZnO:Al synthesized from acac precursors, reflux conditions in benzylamine are recommended over solvothermal treatments in benzylalcohol. Towards further research, these well-identified Al-doped $\mathrm{ZnO}$ nanocrystals will be used as building blocks for the deposition of dense TCO films with good conducting properties.

\section{Acknowledgements}

The reported research was funded by the Research foundation Flanders (FWO Vlaanderen), the Flemish Methusalem Nano program, the SOPPOM program of the Flemish Strategic Initiative for Materials (SIM) and Interreg Vlaanderen Nederland, Solar flare under project number IVA-VLANED1.59. The authors are indebted to G. Reekmans (UHasselt) for the ${ }^{27} \mathrm{Al}-\mathrm{NMR}$ measurements and B. Ruttens (UHasselt) for the X-ray diffraction measurements. We thank A. Abakumov (EMAT, Antwerp University) for fruitful discussions and $\mathbf{M}$. Ieven (UHasselt) for the graphical work.

\section{Notes and references}

1 S. S. Lo, D. Huang, C. H. Tu, C. H. Hou and C. C. Chen, J. Phys. D: Appl. Phys., 2009, 42, 095420.

2 K. J. Chen, T. H. Fang, F. Y. Hung, L. W. Ji, S. J. Chang, S. J. Young and Y. J. Hsiao, Appl. Surf. Sci., 2008, 254, 5791-5795.

3 C. Klingshirn, ChemPhysChem, 2007, 8, 782-803.

4 J. S. Wu and D. F. Xue, Sci. Adv. Mater., 2011, 3, 127-149.

5 M. S. Huh, S. J. Won, B. S. Yang, S. Oh, M. S. Oh, J. K. Jeong and H. J. Kim, J. Vac. Sci. Technol., B: Microelectron. Nanometer Struct.-Process., Meas., Phenom., 2011, 29, 031201.

6 R. Theissmann, S. Bubel, M. Sanlialp, C. Busch, G. Schierning and R. Schmechel, Thin Solid Films, 2011, 519, 5623-5628.
7 D. K. Lee, K. Park, J. H. Ahn, N. E. Lee and Y. J. Kim, J. Nanosci. Nanotechnol., 2011, 11, 5870-5875.

8 I. Gonzalez-Valls, Y. H. Yu, B. Ballesteros, J. Oro and M. Lira-Cantu, J. Power Sources, 2011, 196, 6609-6621.

9 R. C. Pawar, J. S. Shaikh, P. S. Shinde and P. S. Patil, Mater. Lett., 2011, 65, 2235-2237.

10 Y. Bakha, K. M. Bendimerad and S. Hamzaoui, Eur. Phys. J.: Appl. Phys., 2011, 55, 30103.

11 K. W. Liu, M. Sakurai and M. Aono, Sens. Actuators, B, 2011, 157, 98-102.

12 K. Hembram, D. Sivaprahasam and T. N. Rao, J. Eur. Ceram. Soc., 2011, 31, 1905-1913.

13 J. L. He, J. Liu, J. Hu, R. Zeng and W. C. Long, J. Eur. Ceram. Soc., 2011, 31, 1451-1456.

14 J. Clatot, G. Campet, A. Zeinert, C. Labrugere and A. Rougier, Appl. Surf. Sci., 2011, 257, 5181-5184.

15 L. Zhao, G. J. Shao, S. T. Song, X. J. Qin and S. H. Z. Han, Rare Met., 2011, 30, 175-182.

16 S. Cimitan, S. Albonetti, L. Forni, F. Peri and D. Lazzari, J. Colloid Interface Sci., 2009, 329, 73-80.

17 V. L. Kuznetsov and P. P. Edwards, ChemSusChem, 2010, 3, 44-58.

18 R. L. Moss, E. Tzimas, H. Kara, P. Willis and J. Kooroshy, JRC Scientific and Technical Reports, 2011.

19 T. Minami, Semicond. Sci. Technol., 2005, 20, S35-S44.

20 C. Klingshirn, Phys. Status Solidi B, 2007, 244, 3027-3073.

21 E. Fortunato, D. Ginley, H. Hosono and D. C. Paine, MRS Bull., 2007, 32, 242-247.

22 P. Palacios, K. Sanchez and P. Wahnon, Thin Solid Films, 2009, 517, 2448-2451.

23 H. Serier, M. Gaudon and M. Menetrier, Solid State Sci., 2009, 11, 1192-1197.

24 R. Saniz, Y. Xu, M. Matsubara, M. N. Amini, H. Dixit, D. Lamoen and B. Partoens, J. Phys. Chem. Solids, 2013, 74, 45-50.

25 T. Kemmitt, B. Ingham and R. Linklater, J. Phys. Chem. C, 2011, 115, 15031-15039.

26 J. P. Kar, S. Kim, B. Shin, K. I. Park, K. J. Ahn, W. Lee, J. H. Cho and J. M. Myoung, Solid-State Electron., 2010, 54, 1447-1450.

27 M. Bazzani, A. Neroni, A. Calzolari and A. Catellani, Appl. Phys. Lett., 2011, 98, 121907.

28 S. Mridha and D. Basak, J. Phys. D: Appl. Phys., 2007, 40, 6902-6907.

29 J. K. Burdett, G. D. Price and S. L. Price, J. Am. Chem. Soc., 1982, 104, 92-95.

30 K. Schellens, B. Capon, C. De Dobbelaere, C. Detavernier, A. Hardy and M. K. Van Bael, Thin Solid Films, 2012, 524, 81-85.

31 M. Gross, N. Linse, I. Maksimenko and P. J. Wellmann, Adv. Eng. Mater., 2009, 11, 295-301.

32 J. Ederth, P. Heszler, A. Hultaker, G. A. Niklasson and C. G. Granqvist, Thin Solid Films, 2003, 445, 199-206.

33 G. Buhler, D. Tholmann and C. Feldmann, Adv. Mater., 2007, 19, 2224.

34 J. E. Rodriguez-Paez, A. C. Caballero, M. Villegas, C. Moure, P. Duran and J. F. Fernandez, J. Eur. Ceram. Soc., 2001, 21, 925-930.

35 T. Ogi, F. Iskandar, Y. Itoh and K. Okuyama, J. Nanopart. Res., 2006, 8, 343-350.

36 C. H. Lu and C. H. Yeh, Ceram. Int., 2000, 26, 351-357. 
37 L. Cai, G. Jiang, C. Zhu and D. Wang, Phys. Status Solidi A, 2009, 206, 1461-1464.

38 R. Chakravarty and C. Periasamy, Sci. Adv. Mater., 2011, 3, 276-283.

39 E. Hammarberg, A. Prodi-Schwab and C. Feldmann, J. Colloid Interface Sci., 2009, 334, 29-36.

40 T. V. Thu and S. Maenosono, J. Appl. Phys., 2010, 107, 014308.

41 E. Burunkaya, N. Kiraz, O. Kesmez, H. E. Camurlu, M. Asilturk and E. Arpac, J. Sol-Gel Sci. Technol., 2010, 55, 171-176.

42 M. Niederberger, G. Garnweitner, N. Pinna and G. Neri, Prog. Solid State Chem., 2005, 33, 59-70.

43 N. Pinna, G. Garnweitner, M. Antonietti and M. Niederberger, J. Am. Chem. Soc., 2005, 127, 5608-5612.

44 M. Baghbanzadeh, S. D. Skapin, Z. C. Orel and C. O. Kappe, Chem.-Eur. J., 2012, 18, 5724-5731.

45 J. Puetz, N. Al-Dahoudi and M. A. Aegerter, Adv. Eng. Mater., 2004, 6, 733-737.

46 M. T. Weller, Inorganic Materials Chemistry, Oxford University Press, UK, 1996, pp. 15-29.

47 S. Suwanboon, P. Amornpitoksuk, A. Haidoux and J. C. Tedenac, J. Alloys Compd., 2008, 462, 335-339.

48 Y. S. Avadhut, J. Weber, E. Hammarberg, C. Feldmann and J. Gunne, Phys. Chem. Chem. Phys., 2012, 14, 11610-11625.
49 K. J. D. MacKenzie and M. E. Smith, Multinuclear Sold-State NMR of Inorganic Materials, Elsevier Science Ltd, UK, 2001, pp. 271-300.

50 J. U. Brehm, M. Winterer and H. Hahn, J. Appl. Phys., 2006, 100, 064311.

51 Y. L. Zhang, Y. Yang, J. H. Zhao, R. Q. Tan, W. Y. Wang, P. Cui and W. J. Song, J. Mater. Sci., 2010, 46, 774-780.

52 E. Bacaksiz, S. Aksu, S. Yilmaz, M. Parlak and M. Altunbas, Thin Solid Films, 2010, 518, 4076-4080.

53 P. Sagar, M. Kumar and R. M. Mehre, Thin Solid Films, 2005, 489, 94-98.

54 R. Buonsanti, A. Llordes, S. Aloni, B. A. Helms and D. J. Milliron, Nano Lett., 2011, 11, 4706-4710.

55 F. Rueda, J. Mendialdua, A. Rodriguez, R. Casanova, Y. Barbaux, L. Gengembre and L. Jalowiecki, J. Electron Spectrosc. Relat. Phenom., 1996, 82, 135-143.

56 M. Chen, X. Wang, Y. H. Yu, Z. L. Pei, X. D. Bai, C. Sun, R. F. Huang and L. S. Wen, Appl. Surf. Sci., 2000, 158, 134-140.

57 M. Chen, Z. L. Pei, C. Sun, L. S. Wen and X. Wang, Mater. Lett., 2001, 48, 194-198.

58 H. B. Fan, S. Y. Yang, P. F. Zhang, H. Y. Wei, X. L. Liu, C. M. Jiao, Q. S. Zhu, Y. H. Chen and Z. G. Wang, Chin. Phys. Lett., 2007, 24, 2108-2111. 